\title{
Intelligenter Zahnriemen
}

\author{
Dominik Großkurth ${ }^{1}$, Georg Martin ${ }^{2}$ \\ ${ }^{1}$ Fachgebiet Integrierte Elektronische Systeme, TU Darmstadt, Merckstraße 25, 64283 Darmstadt \\ ${ }^{2}$ Fachgebiet Produktentwicklung und Maschinenelemente, TU Darmstadt, Otto-Berndt-Straße 2, \\ 64287 Darmstadt
}

\section{Zusammenfassung}

In dieser Arbeit wird ein Messsystem beschrieben, mit dem es möglich ist Messwerte innerhalb eines Zahnriemens zu erheben und diese, während des Betriebs des Zahnriemens, an eine externe Stelle zu senden.

Ein solches Messsystem ermöglicht die Zustandsüberwachung des Zahnriemens im Betrieb und ist damit ein weiterer Schritt hin zu einer vollständig vernetzten Produktion. Das Hauptaugenmerk wird dabei auf den Verschleiß des Zahnriemens gelegt, da dieser aktuell nur umständlich durch Personal vor Ort ermittelt werden kann. Als Indikator dienen dabei die Vorspannkraft des Zahnriemens, welche indirekt aus seiner Eigenfrequenz ermittelt wird, und die Temperatur im Inneren des Zahnriemens. Zum Aufnehmen dieser beiden Messgrößen wurde ein Sensorknoten entwickelt, der klein genug ist, um in einen Zahn des Zahnriemens eingesetzt werden zu können.

Erste Messungen bestätigen, dass es möglich ist mittels Betriebsschwingungsdaten Rückschlüsse auf die Vorspannkraft und damit auf den Verschleiß des Zahnriemens zu ziehen.

Zudem wird die mechanische Belastung des Sensorknotens näher untersucht und eine verbesserte Version vorgestellt. Die neue Version benötigt signifikant weniger Bauraum und kann voraussichtlich besser mit den beschrieben Belastungen umgehen.

Keywords: Zustandsüberwachung, Zahnriemen, Schwingungsmessung

\section{Problemstellung}

In Industrieanlagen und Produktionsmaschinen wird im Rahmen von Industrie 4.0 zunehmend eine zustandsorientierte Instandhaltung umgesetzt, die auf der Zustandsüberwachung (Condition Monitoring) von Bauteilen beruht. Dies ermöglicht es, Wartungskosten zu reduzieren und ungeplante Maschinenausfälle zu vermeiden.

In vielen industriellen Anlagen werden Zahnriemengetriebe mit Hochleistungszahnriemen aus Polyurethan eingesetzt, um Bewegungen zu übertragen. Sie bestehen typischerweise aus Zugträgern, meist Stahlseile, und einer Matrix aus Polyurethan, die auch die Zähne bildet, vgl. Abbildung 1.

Im einfachsten Fall besteht ein Zahnriemengetriebe aus einer Antriebsscheibe und einer Abtriebsscheibe, die vom Zahnriemen umschlungen werden. Die Betriebskraft wird in den Zahnscheiben über die PU-Matrix in den Riemen übertragen, im Riemenabschnitt zwischen zwei Scheiben (sog. Trum) wird die Kraft hauptsächlich durch die Zugträger geleitet.

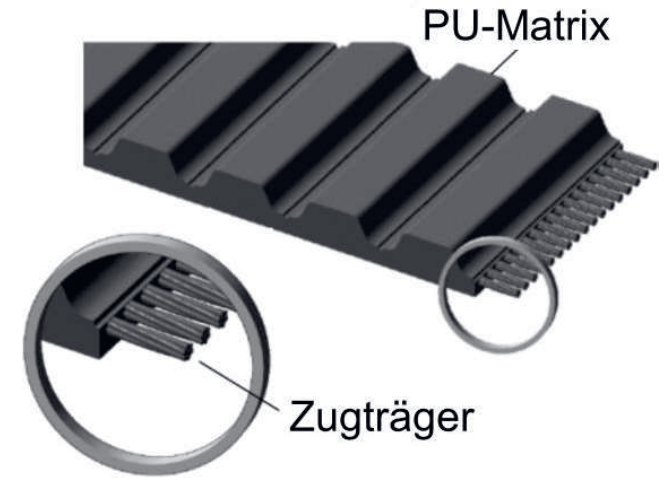

Abbildung 1. Aufbau eines PolyurethanZahnriemens, Abb. nach [1]

Ein Zahnriemen benötigt prinzipbedingt eine Vorspannkraft $F_{\mathrm{TV}}$, um effektiv und effizient zu funktionieren, vgl. Abbildung 2. Bei zu geringer Vorspannkraft findet der Zahneingriff in der Riemenscheibe nicht mehr korrekt statt, was zu Schwingungen im Riemen und der umgebenden Maschine sowie zu erhöhtem Verschleiß führt. 


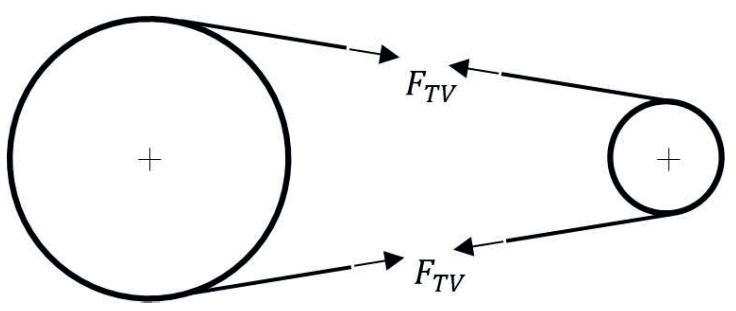

Abbildung 2. Vorspannkraft in einem Zahnriemengetriebe

Im Verlauf der Betriebszeit verschleißen die Zugträger, infolgedessen nimmt die Steifigkeit des Zahnriemens ab. Dies führt zu einer Abnahme der Vorspannkraft, einer verschlechterten Laufruhe und einem erhöhten Verschleiß, sodass bei starkem Zugträgerverschleiß der Zahnriemen ausgetauscht werden muss [2]. Wenn der Riemen nicht rechtzeitig getauscht wird, kann der Zugträgerverschleiß fortschreiten, bis es zum Riss des Zahnriemens und damit zum Ausfall der gesamten Maschine kommt.

Der Zugträgerverschleiß begrenzt in vielen industriellen Anwendungen die Lebensdauer von PU-Zahnriemen. Allerdings existiert kein Modell zur Berechnung der Lebensdauer eines Zahnriemengetriebes, wie dies z.B. für Stirnradgetriebe etabliert ist. Aufgrund dieser Unsicherheit bezüglich der Lebensdauer treten einerseits immer wieder ungeplante Ausfälle auf, andererseits werden viele Zahnriemen vor Erreichen ihres tatsächlichen Lebensdauerendes vorsorglich getauscht. Eine Zustandsüberwachung, welche das Erreichen eines kritischen Verschleißmaßes erkennt, kann deshalb einen wichtigen Beitrag zu einer kosten- und ressourceneffizienteren Produktion leisten. Allerdings sind bisher, anders als z.B. für Wälzlager, noch keine Zustandsüberwachungssysteme für Zahnriemen verfügbar. Die Überwachung des Riemenzustands kann bisher nur durch die manuelle Kontrolle der Vorspannkraft erfolgen.

Gemessen wird diese Kraft, indem der Riemen durch manuelles Anschlagen breitbandig zu Schwingungen angeregt wird und aus der Schwingungsantwort die Eigenfrequenzen bestimmt werden. Die Eigenfrequenzen $f_{\lambda}$ sind, wie bei einer Gitarrensaite, durch die Beziehung

$$
f_{\lambda}=\lambda \cdot \sqrt{\frac{F_{T V}}{4 \cdot m^{\prime} \cdot l^{2}}}
$$

mit der Vorspannkraft verknüpft. Dabei ist $m^{\prime}$ das Längengewicht in $\mathrm{kg} / \mathrm{m}, l$ die frei schwingende Trumlänge zwischen zwei Scheiben und $F_{\mathrm{TV}}$ die Vorspannkraft. $\lambda$ bezeichnet die Ordnung der Eigenfrequenz, d.h. $\lambda=1$ bezeichnet die erste Eigenfrequenz mit einem Schwin- gungsbauch, $\lambda=2$ die zweite Eigenfrequenz mit zwei Schwingungsbäuchen und einem Schwingungsknoten, etc. Dieses Messverfahren ist etabliert und gilt als das VorspannkraftMessverfahren mit der höchsten Genauigkeit [1]. Entsprechende Messgeräte sind von diversen Herstellern auf dem Markt verfügbar.

Die regelmäßige manuelle Kontrolle der Vorspannkraft ist aufgrund des Personalaufwands und der schlechten Zugänglichkeit in den meisten Fällen nicht möglich. An der TU Darmstadt wird deshalb in einem Kooperationsprojekt mit BRECO Antriebstechnik Breher $\mathrm{GmbH}$ \& Co. KG und CONTECS Engineering Services $\mathrm{GmbH}$ ein Zustandsüberwachungssystem entwickelt, das auf einer schwingungsbasierten Vorspannkraftmessung ohne externe Anregung, nur anhand der Betriebsschwingungen, basiert. Diese Zustandsüberwachung soll nicht nur ungeplante Ausfälle verhindern, sondern auch eine optimierte und damit kosteneffizientere Auslegung des Riemengetriebes ermöglichen, sowie Folgeschäden an der Maschine oder eine mangelhafte Qualität von Fertigungsprozessen vermeiden, indem die Laufruhe des Riementriebs sichergestellt wird.

\section{Lösungsvorschlag}

Zur Messung von Parametern, die potentiell auf den Verschleiß eines Zahnriemens hinweisen, wurde daher ein Sensorknoten entwickelt. Dieser ermöglicht das Erfassen von Beschleunigungen sowie der Temperatur und ist in der Lage diese Messwerte drahtlos an eine Basisstation zu übertragen.

Dabei wird die Beschleunigung an zwei möglichst weit voneinander entfernten Punkten $(35 \mathrm{~mm})$ in allen drei Raumrichtungen aufgenommen. Die Position der Beschleunigungsmesser auf der Platine ist in Abbildung $4 \mathrm{zu}$ sehen.

Zur besseren Beschreibung der Schwingungsrichtungen wird ein Koordinatensystem für den Zahnriemen definiert, zu sehen in Abbildung 3 , auf das sich im Folgenden bezogen wird. Mithilfe der Beschleunigungssensoren ist es möglich die Transversalschwingungen des Zahnriemens zu messen. Diese treten in z-Richtung auf und werden durch den Polygon-Effekt angeregt. Dieser Effekt tritt auf wenn ein Zugmittel über ein Antriebsrad formschlüssig angetrieben wird. Zugmittel wie Zahnriemen oder Ketten bestehen aus einzelnen verhältnismäBig starren Segmenten, wodurch sie nicht kreisrund auf- bzw. ablaufen können. Hierdurch verhält sich das runde Antriebsrad wie ein Polygon dessen Seitenzahl von dessen Durchmesser und der Größe der Segmente des Zugmittels abhängt. Durch die Polygonform der Zahnscheiben ist der wirksame Radi- 
us, mit dem der Zahnriemen um die Scheibe läuft, während einer Umdrehung nicht konstant. Deshalb variiert, auch bei gleichförmiger Winkelgeschwindigkeit, die Geschwindigkeit des Zahnriemens und sein Abstand zur Scheibenachse. Diese Ungleichförmigkeiten regen u.a. transversale Schwingungen des Riemens an.

Des Weiteren können Stöße beim Anlaufen an die Bordscheiben erkannt werden, da diese maßgeblich in $y$ - Richtung auftreten. In $x$ Richtung können Stöße des Antriebs und des Abtriebes erkannt werden, die die anderen Messungen stören können.

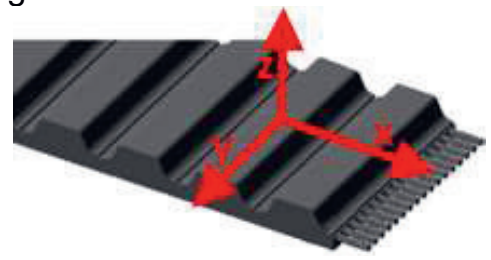

Abbildung 3. Koordinatensystem bezogen auf einen Punkt auf dem Zahnriemen

Der zweite Beschleunigungssensor dient dazu, Torsionsschwingungen des Zahnriemens um die Achse der Laufrichtung (x-Achse) erkennen zu können, um zu verhindern, dass Torsionseigenfrequenzen fälschlicherweise als SaitenEigenfrequenzen interpretiert werden. Durch diese Torsionsschwingungen käme es zu einer sich periodisch ändernden Phasenverschiebung der z-Beschleunigungssignale der beiden Sensoren, während reine Transversalschwingungen durch gleichphasige Signale charakterisiert sind. Aufgenommen werden die Beschleunigungen mittels eines kompakten und Energie sparenden MEMS Beschleunigungssensors. Dieser erfasst Beschleunigungen mit einer Amplitude von bis $\mathrm{zu} \pm 400 \mathrm{~g}$, löst diese mit 12Bit auf und stellt sie digitalisiert über eine SPI Schnittstelle zur Verfügung.

Die Zustandsüberwachung basiert darauf, dass ein Zahnriemen beim Zahneingriff zu Schwingungen angeregt wird. Dabei wirken mehrere Anregungsmechanismen, hervorzuheben ist die Anregung durch den Polygoneffekt. Wenn die Schwingungsanregung im Betrieb breitbandig ist, kann die SaitenEigenfrequenz anhand dieser gemessenen Betriebsschwingungen bestimmt werden.

Kommt es durch Verschleiß zur Längung des Zahnriemens, so stimmt der Abstand der Zähne nicht länger mit dem Abstand der Lücken auf der Zahnscheibe überein. Während dieses Prozesses erhöht sich allmählich die Reibung der Zahnflanken an den Zahnscheiben und führt so zu einer steigenden Erwärmung des Zahnriemens. Aus diesem Grund wurde als zweite Messgröße die Temperatur ausgewählt. Aufgenommen wird sie durch einen separaten
Sensor, dieser ermöglicht die Messung von Temperaturen von $-40^{\circ} \mathrm{C}$ bis $+125^{\circ} \mathrm{C}$, löst diese mit 12 Bit auf und kann über eine $12 \mathrm{C}$ Schnittstelle ausgelesen werden.

Als Mikrocontroller wurde der BGM121 [3] der Firma Silicon Labs eingesetzt. Dieser ist ein System in Package (SiP), er setzt auf einen ARM Cortex-M4 und enthält bereits passive Komponenten sowie eine integrierte Antenne über die er mittels Bluetooth Low Energy mit der Außenwelt kommunizieren kann. Dies ist nötig, da der Zahnriemen im Betrieb mit hohen Geschwindigkeiten rotiert und nicht drahtgebunden kontaktiert werden kann. Aus demselben Grund wurde für die Energieversorgung während der Messungen ein Li-Polymer-Akku eingesetzt.

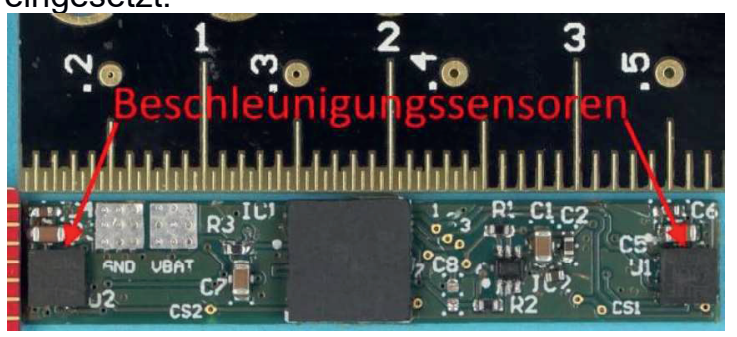

Abbildung 4. Sensorknoten zur Schwingungsmessung innerhalb von Zahnriemen

Abbildung 4 zeigt die bestückte Platine des Sensorknotens. An den äußeren Rändern befinden sich die beiden Beschleunigungssensoren, als größte Komponente ist der Mikrocontroller in der Mitte angeordnet und rechts neben inm befindet sich der sechsbeinige Temperatursensor.

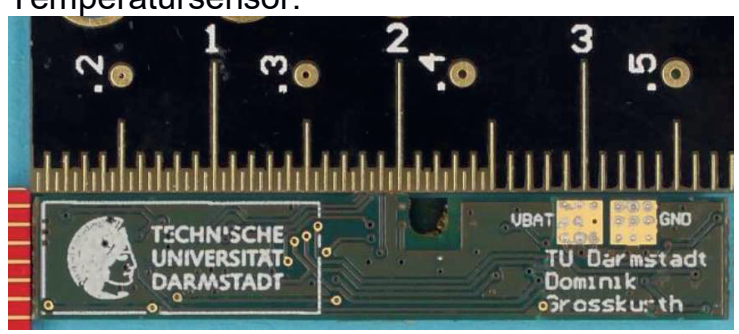

\section{Abbildung 5. Unterseite des Sensorknotens}

Als größte Herausforderung stellte sich die Unterbringung des Sensorknotens innerhalb des Zahnriemens heraus. Da der Wunsch bestand, Zahnriemen auch auf Prüfständen mit Rückenrolle einsetzen zu können, konnten keine Komponenten auf der Rückseite des Riemens angebracht werden. Damit verblieb als einzige Option die Einbringung innerhalb eines Zahnes. Daher wurde eine etwa $4 \times 7 \times 47 \mathrm{~mm}$ große Aussparung in zwei Zähne des Zahnriemens gefräst, um in einem der Zähne den Sensorknoten und in dem anderen Zahn den Li-Polymer-Akku unterzubringen. Verbunden wurden beide Zähne mit Leitern, 
die nachträglich im Zwischenraum der Zugträger verlegt wurden. Verfüllt wurde der mit dem Sensorknoten bestückte Zahn mit einem Schmelzklebstoff aus Ethylen-VinylacetatCopolymer (EVA), da dieser eine ähnliche Steifigkeit und Verarbeitungstemperatur wie das Matrixmaterial Polyurethan der Zahnriemen aufweist. In Abbildung 6 ist die so vergossene Elektronik zu sehen, bevor das überschüssige Vergussmaterial entfernt wurde.

Eine Vorverarbeitung der Sensorsignale innerhalb des Sensorknotens ist nicht gewünscht, so ergibt sich die nötige Datenrate aus den drei Achsen der zwei Beschleunigungssensoren und deren Abtastrate. Die Messwerte werden zur leichteren Handhabung auf 16Bit erweitert und die Abtastrate wird auf $800 \mathrm{~Hz}$ festgelegt, da mit Frequenzen $<200 \mathrm{~Hz}$ gerechnet wird.

Zum Erreichen einer möglichst großen Datenrate wurde die Paketgröße der Funkübertragung von standardmäßig 23Byte auf 241Byte erhöht und das Verbindungsintervall auf $7,5 \mathrm{~ms}$ reduziert. Somit war eine stabile Übertragung von allen Sensorwerten gewährleistet $(2 \cdot 3 \cdot 16 \mathrm{Bit} \cdot 800 \mathrm{~Hz}=76,8 \mathrm{kBit} / \mathrm{s})$.

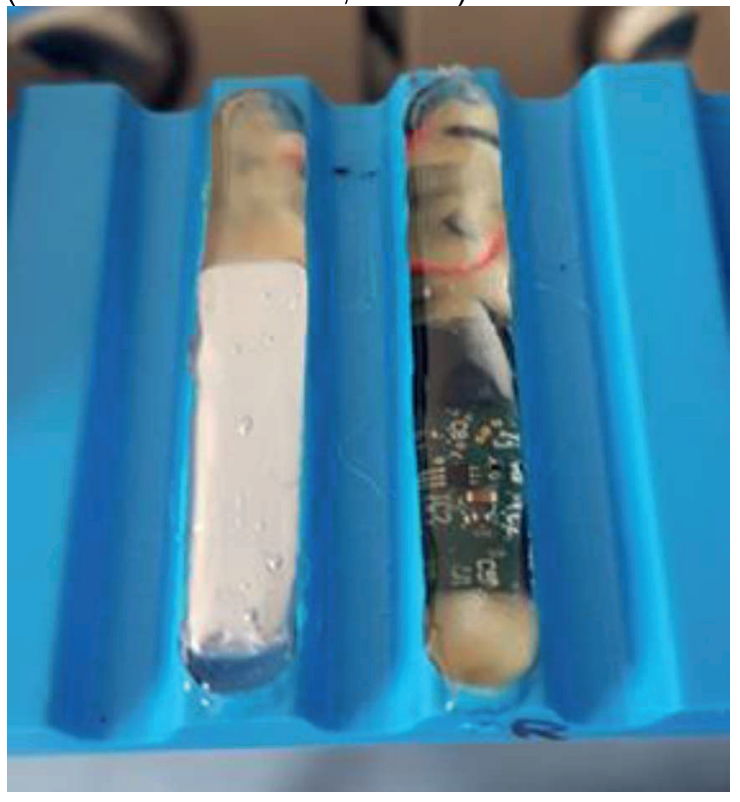

Abbildung 6. In den Zahnriemen eingebetteter Sensorknoten und Energiespeicher

Empfangen werden die Daten ebenfalls von einem Mikrocontroller der Firma Silicon Labs, dieser übermittelt die Daten anschließend per USB an einen Computer, wo die Daten von einem Programm entgegengenommen werden. Dieses Programm ermöglicht dem Anwender die ankommenden Daten als FFT-Plot zu sehen oder die Rohdaten abzuspeichern. Außerdem ist eine Konfiguration der Beschleunigungssensoren möglich. Sie bieten die Möglichkeit den Messbereich zu ändern
$( \pm 400 \mathrm{~g}, \pm 200 \mathrm{~g}, \pm 100 \mathrm{~g})$, um kleinere Amplituden präziser auflösen zu können und zudem einen internen Hochpassfilter zuzuschalten $(0 \mathrm{~Hz}, 2,5 \mathrm{~Hz}, 5 \mathrm{~Hz}, 10 \mathrm{~Hz}, 20 \mathrm{~Hz})$.

\section{Experimentelle Untersuchungen}

Um die grundsätzliche Eignung einer riemenintegrierten Schwingungsmessung für die $\mathrm{Zu}$ standsüberwachung zu überprüfen, wurde ein Versuch aufgebaut. Dabei wurden zwei Fragestellungen untersucht: Erstens sollte geprüft werden, ob die Schwingungsanregung so breitbandig ist, dass die Eigenfrequenzen im Spektrum sichtbar sind. Zweitens sollte die Haltbarkeit der Sensorelektronik unter den mechanischen Beanspruchungen, insbesondere durch die Zentripetalkraft und die Biegung beim Scheibendurchlauf, untersucht werden.

Der Versuchsaufbau besteht aus einem einfachen Riementrieb mit zwei Scheiben. Eine Scheibe ist angetrieben, die zweite Scheibe stellt lediglich eine Umlenkung ohne anliegendes Drehmoment dar. Der beschriebene Versuchsaufbau wurde zum einem vor Ort an der TU Darmstadt aus Aluminium-SystemProfilelementen aufgebaut und entspricht einer Maschine mit geringer Steifigkeit. Zum anderem wurde ein Prüfstand der Firma BRECO Antriebstechnik verwendet der sowohl eine hohe Masse als auch eine hohe Steifigkeit aufweist.

Während der ersten Messungen traten nach wenigen Minuten Verbindungsabbrüche auf. Nachdem ausgeschlossen wurde, dass Metallteile die Funkstrecke blockieren, wurde der Riemen gezielt mechanischen Belastungen in verschiedenen Richtungen ausgesetzt. Hierbei zeigte sich, dass die Verbindungsprobleme bei Torsion des Zahnriemens oder Biegen entlang eines Zahnes (y-Richtung) auftreten. Die Platine wurde anschließend außerhalb eines Riemens diesen Belastungen ausgesetzt. Es stellte sich heraus, dass sich die Platine gleichmäßig über ihre gesamte Länge biegt. Das mit $6,5 \mathrm{~mm}$ Kantenlänge verhältnismäßig lange System in Package (SiP, BGM121) verformt sich bedingt durch sein aus Duroplast bestehendes Gehäuse jedoch nicht. Dies führt zu einer mechanischen Belastung der Lötstellen unterhalb des SiP und zu deren Bruch. In Abbildung 9 sind diese Stellen, sowie potentiell problematische Stellen an den Beschleunigungssensoren markiert. Abbildung 7 zeigt ein Foto eines gebogenen Sensorknotens. Die Biegung entsteht hierbei durch das aufbringen einer Kraft an den Enden der Platine mittels einer Klemmvorrichtung. Eine Vergrößerung des kritischen Abschnitts ist in Abbildung $8 \mathrm{zu}$ sehen. 


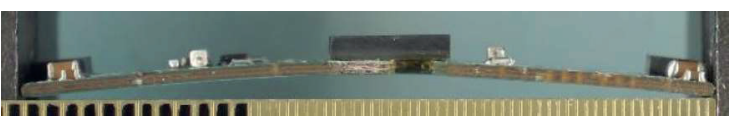

\section{Abbildung 7. Durch Einspannen verformter Sensorknoten}

Die Ursprüngliche Vergussmasse aus EVA weist eine relativ hohe Steifigkeit auf und zwingt den Sensorknoten sich im gleichen Maße wie den Zahnriemen zu verbiegen. In weiteren Versuchen wurde daher der Zahn mit weicherem Silikon (1-K-Polysiloxan) vergossen, um die mechanischen Belastungen zu reduzieren. Zudem wurden die Stellen an denen die Beschleunigungssensoren und das SiP platziert sind mit Epoxidharz versteift. Auch hier erwiesen sich die mechanischen Belastungen im Betrieb als zu hoch.

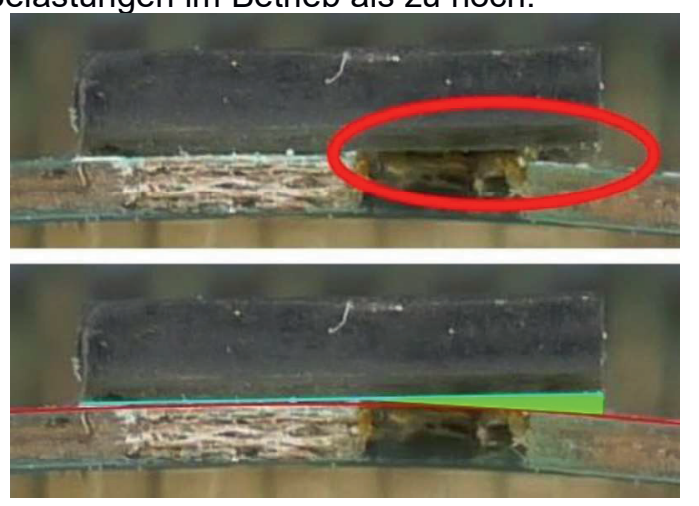

Abbildung 8. Bruch der Lötstellen unterhalb des System in Package

Verstärkt wurde diese Problematik durch die Schwächung der Platine unterhalb des Mikrocontrollers. Diese wurde vorgenommen, um die Abstrahlcharakteristik der darüber liegenden Antenne im SiP nicht zu verändern. Abbildung 5 zeigt die Elektronik von unten, dort ist die Schwächung zwischen der $2 \mathrm{~cm}$ und der $2,2 \mathrm{~cm}$ Markierung an der oberen Seite zu erkennen.

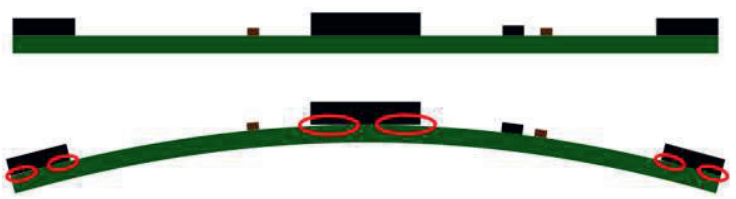

Abbildung 9. Belastung der Lötstellen des Sensorknotens während des Betriebs

Erst das Fixieren des Sensorknotens am Zahngrund mit einem flexiblen Kontaktklebstoff (Polychloropren) und der Verzicht auf das Verfüllen des Zahns führte dazu, dass über mehrere Stunden stabil gemessen werden konnte.

Abbildung 10 zeigt das Spektrogramm eines Hochlaufs bis $340 \mathrm{U} / \mathrm{min}$. Deutlich sichtbar ist, dass das Schwingungsverhalten insgesamt von der Zahneingriffsfrequenz und ihren höheren Ordnungen dominiert wird. Aber auch die erste Eigenfrequenz und höhere Eigenfrequenzen bis zur 6 . Ordnung $(\lambda=6)$ werden über den gesamten Drehzahlbereich angeregt. In den Resonanzdurchfahrten, d.h. wenn die Zahneingriffsfrequenz einer Eigenfrequenz entspricht, treten deutliche Amplitudenüberhöhungen auf. Diese Ergebnisse deuten darauf hin, dass im Betrieb eine ausreichend starke und breitbandige Schwingungsanregung stattfindet, um die Eigenfrequenz des Riemens zu bestimmen.

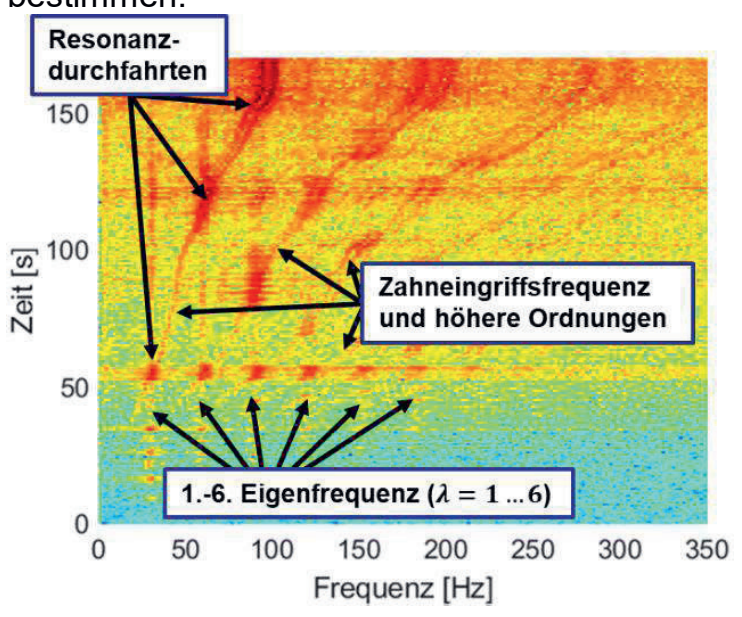

Abbildung 10. Spektrogramm des sensorintegrierten Beschleunigungssensors bei einem Hochlauf

\section{Ausblick}

Mit diesen Ergebnissen wurde eine neue Version des Sensorknotens entwickelt. Bei dieser wird auf einen zweiten Beschleunigungsmesser verzichtet. Zudem wird ein Mikrocontroller verwendet, der keine integrierte Antenne besitzt und im Wafer Level Package vorliegt (WLCP). So kann die Größe der Elektronik von $6,5 \times 37 \times 2 \mathrm{~mm} \approx 480 \mathrm{~mm}^{3}$ auf $3,2 \times 25 \times 1,75 \mathrm{~mm} \approx$ $130 \mathrm{~mm}^{3}$ um den Faktor 3,7 reduziert werden. Abbildung 11 zeigt den neuen Sensorknoten eingelegt in einen AT10 Zahnriemen der Firma BRECO Antriebstechnik. Dies ermöglicht die Unterbringung in kleineren Zähnen, wodurch es möglich wird kleinere Zahnriementypen zu untersuchen. 


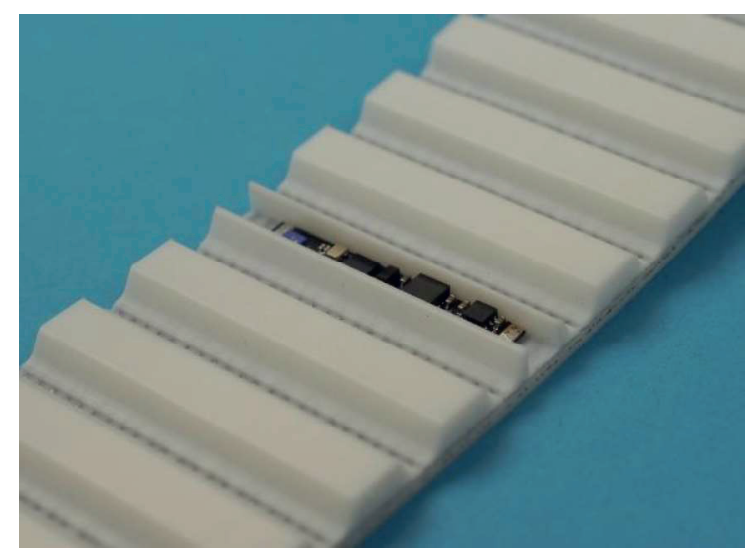

Abbildung 11. Neuer Sensorknoten in einem AT10 Zahnriemen der Firma BRECO Antriebstechnik

Außerdem wurde bei dieser Version eine $0,2 \mathrm{~mm}$ dicke, flexible Leiterplatte aus Polyimid verwendet. Die Bauteile wurden dabei in kleinen Gruppen angeordnet, sodass unterhalb der Bauteile jeweils eine $0,5 \mathrm{~mm}$ dicke Versteifung aus FR4 angebracht werden kann, um diese Bereiche vor mechanischen Belastungen zu schützen. In Abbildung 12 ist ein Foto mit einer verformten, unbestückten Platine zu sehen bei der die entsprechenden Biegestellen markiert wurden.

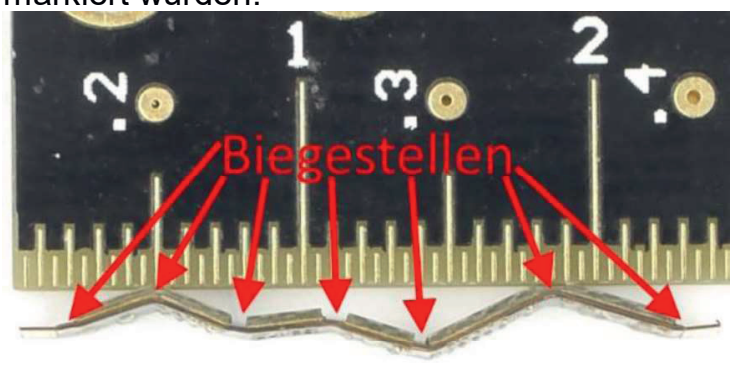

Abbildung 12. Flexible Leiterplatte mit Versteifungen

Mittels dieser Elektronik müssen nun weitere Versuche in verschiedenen Produktionsanlagen stattfinden, um die mechanische Stabilität dieses Ansatzes zu verifizieren.

Die bei diesen Versuchen gewonnen Messergebnisse dienen im weiterem zur Verifikation der Annahme, dass der Verschleiß des Zahnriemens mit an der Abnahme der Vorspannkraft abgelesenen werden kann und dass diese indirekt über die Eigenfrequenz, während des Betriebes ermittelt werden kann.

\section{Literaturnachweis}

[1] T. Nagel, Zahnriemengetriebe: Eigenschaften, Normung, Berechnung, Gestaltung. München: Hanser, 2008.
[2] R. Perneder, Handbuch Zahnriementechnik: Grundlagen, Berechnung, Anwendungen. Berlin: Springer, 2009.

[3] „BGM121/BGM123 Blue Gecko Bluetooth SiP Module Data Sheet". Silicon Laboratories Inc. 